\title{
Krankheiten erriechen? Eine kurze Übersicht über elektronische Nasen
}

\author{
Smelling Diseases? A Short Review on Electronic Noses
}

Autoren

Institute
A. R. Koczulla' ${ }^{1}$, A. Hattesohl', H. Biller ${ }^{2}$, J. Hofbauer ${ }^{3}$, J. Hohlfeld², C. Oeser ${ }^{4}$, C. Gessner ${ }^{4}$, C. Vogelmeier' ${ }^{1}$, J. I. Baumbach ${ }^{5}$, H. Wirtz ${ }^{4^{*}}$, R. A. Jörres ${ }^{3}$

Die Institutsangaben sind am Ende des Beitrags gelistet. eingereicht 23.12.2010 akzeptiert nach Revision 1.2.2011

\section{Bibliografie}

Dol http://dx.doi.org/ $10.1055 / \mathrm{s}-0030-1256252$

Online-Publikation: 16.3.2011

Pneumologie 2011; 65:

401-405 @ Georg Thieme

Verlag KG Stuttgart · New York ISSN 0934-8387

\section{Korrespondenzadresse}

Dr. A. R. Koczulla

Universitätsklinikum Gießen und Marburg

Abteilung für Pulmonologie

Standort Marburg

Baldingerstraße 1

35042 Marburg

koczulla@med.uni-marburg.de

\section{Zusammenfassung $\nabla$}

Nichtinvasive Atemwegsuntersuchungen stellen eine vielversprechende Entwicklung in der pulmonalen Diagnostik der letzten 15 Jahre dar. Neben der Messung des exhalierten Stickstoffmonoxids (FeNO) werden für wissenschaftliche Fragestellungen vermehrt breiter gespannte, technisch diffizilere Verfahren wie elektronische Nasen (EN) verwendet. EN zielen darauf ab, das menschliche Geruchsorgan teilweise zu imitieren. Verschiedene Typen von EN werden zurzeit für wissenschaftliche Fragestellungen getestet. Neben Geräten, die auf Messung der Ionenmobilität beruhen, oder Massenspektrometern werden Geräte verwendet, die auf einer Auswahl von PolymerSensoren beruhen, die volatile organische Komponenten (VOCs) abhängig von Größe, Struktur, Polarität und Protonenaffinität binden und so kombinierte Signalmuster erzeugen, ohne dass in der Regel Einzelkomponenten identifiziert werden. Die Analysen erfolgen dabei über eine Mustererkennung. Einige Verfahren, zum Beispiel die Ionenmobilitätspektroskopie (IMS) oder Massenspektroskopie (MS), ermöglichen auch eine direkte Bestimmung von Komponenten. Vielfach sind allerdings die substanzspezifischen Muster noch nicht für alle vorkommenden Analyte in Datenbanken hinterlegt, sodass auch hier häufig zur Differenzierung eine Mustererkennung verwendet wird. In der Medizindiagnostik stehen der nichtinvasive Zugang und die „onsite“ Verfügbarkeit dieser Verfahren im Vordergrund. Von einem Routineeinsatz ist man noch entfernt, jedoch weisen die Ergebnisse auf vielversprechende Anwendungsmöglichkeiten hin. In der folgenden Übersicht wollen wir daher die Verfahren kurz auf dem derzeitigen Wissensstand vorstellen.

\section{Abstract \\ $\nabla$}

Non-invasive pulmonary diagnostics is a promising and interesting field in respiratory medicine. Beside exhaled breath condensate, there is an increasing interest in alternative and faster techniques such as electronic noses (EN). EN aim to mimic or improve the sense of smelling. Different types of EN have been employed in research so far. In addition to ion mobility spectrometry and mass spectrometry, ENs that consist of various biopolymer sensors for the sensing of volatile organic compounds (VOCs) have been tested. VOCs bind to the sensors depending on size, structure, hydrogen binding and polarity. This leads to physical alterations, e.g., swelling resulting in a change of resistance. The smell print represents composite patterns in contrast to single compounds, and the distinction between different categories is achieved by pattern recognition algorithms. Other types of EN like mass spectrometry and ion mobility spectrometry are capable of identifying even single analyte fractions provided that their characteristics have been saved in data repositories. The non-invasive nature, onsite availability and relatively cheap sampling are advantages of ENs that underly the increasing interest in their use for medical purposes. Some promising results have already been published. This review aims to describe the state of the art in brief form.

\footnotetext{
* Herr Wirtz und Herr Jörres haben äquivalenten Anteil an der Arbeit.
} 
32 Dünnschicht-Kohlenstoff-Polymer-Chemiresistoren jedes Polymer anders zusammengesetzt

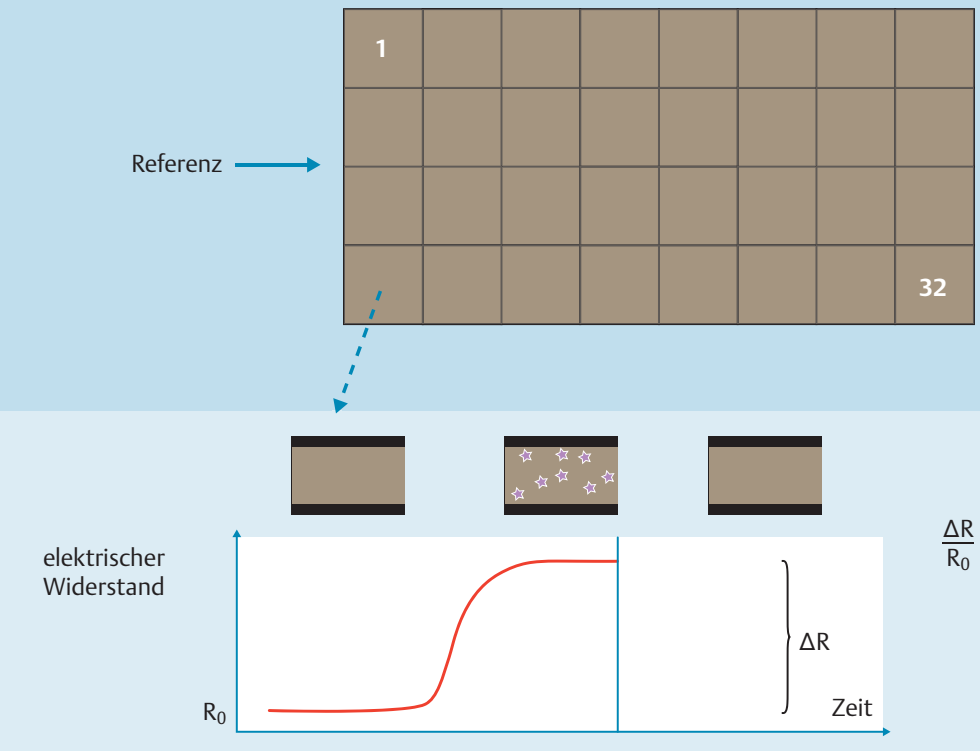

Abb. 1 Funktionsweise der in der Literatur bislang am häufigsten verwendeten elektronischen Nase Cyranose $320^{R}$.

\section{Bauprinzipien von elektronischen Nasen}

Klassische EN umfassen ein Array von elektronischen Sensoren. Alternative Verfahren stellen u. A. Gas-Chromatografie, Massenspektrometrie (MS) und Ionen-Mobilitäts-Spektrometrie (IMS) dar, die weiter unten beschrieben werden.

Die Aufgabe der Sensoren ist die Wandlung eines chemischen Signals in ein elektrisches Signal, das der Summe der gasförmigen Moleküle oder Ionen entspricht, gegen die der Sensor empfindlich ist $[1,2]$. Die Sensoren der elektronischen Nase sprechen auf unterschiedliche Masse, Ladung und/oder andere Eigenschaften von Molekülen an, z. B. deren Polarität oder bestimmte chemische Gruppen. Verschiedene Typen von Sensoren wurden bislang in EN implementiert:

1. Piezoelektrische Sensoren (gravimetrisch/akustisch).

2. (Elektro-)chemische Sensoren

3. Optische Sensoren

4. Kalorimetrische oder thermale Sensoren

5. Biopolymere

Die ersten EN bestanden aus einem Array von Sensoren des gleichen Typs. Experimente zeigten jedoch, dass vor allem in einer komplexen Matrix nicht immer genügend viele Informationen für Detektion und Unterscheidung generiert werden konnten. Nachfolgend wurden Kombinationen von Sensoren (exemplarisch Cyranose $320^{\mathrm{TM}}$, $\odot$ Abb. 1) verwendet und sogenannte Hybridsysteme entwickelt. Das bedeutete zum einen eine komplexere Elektronik, zum anderen ergaben sich Probleme mit der Standardisierung dieser Systeme, die bis heute fortbestehen $[3,4]$.

\section{Datenverarbeitung \\ $\nabla$}

Die Analyse der Daten beruht auf einer statistisch basierten Mustererkennung (häufig „Pattern Recognition“ genannt). Formal erfolgt die Erstellung einer Tabelle (Matrix) anhand der Signalmuster (z. B. spaltenweise die Sensoren und zeilenweise die Proben). Ein Vektor, der aus den Daten als gemeinsames Merkmal gene- riert wird (im einfachsten Fall der Mittelwert der Zeilen = Proben), wird dann verwandt, um die Ähnlichkeit mit vorgespeicherten Mustern zu analysieren oder neue Muster hinzuzufügen. Sinnvollerweise werden die Daten vorverarbeitet im Sinne einer Standardisierung, möglichen Vorauswahl als relevant bekannter Variablen oder einer statistischen Aufkonzentration der Daten. Letzteres bedeutet, dass die Information einer Vielzahl von Sensoren anhand ihrer Korrelation in wenige virtuelle Variablen zusammengefasst wird und damit eine Reduktion der Dimension der Daten erfolgt $(\bullet$ Abb. 2).

Die Hauptkomponentenanalyse (PCA) und artifizielle neuronale Netzwerke (ANN) stellen die bislang am häufigsten verwendeten Methoden der Datenanalyse dar.

\section{Hauptkomponentenanalyse (PCA)}

Hierbei handelt es sich um eine Methode, die Dimension eines Datensatzes auf optimale Weise zu reduzieren $(\boldsymbol{\Theta}$ Abb.2). Ziel ist es, die Zusammenhänge zwischen den Variablen dazu zu verwenden, neue, hypothetische, statistisch abgeleitete Variablen zu definieren, welche alle wesentliche Information der gemessenen Variablen beinhalten. Zu diesem Zweck versucht man, zuerst diejenige lineare Kombination der Variablen (Komponente) zu finden, welche die Varianz der Messwerte maximal umfasst, und dann schrittweise diejenigen Komponenten, die den jeweils verbleibenden größten Anteil der Varianz abbilden. Im Idealfall gelangt man auf diese Weise zu einer kleinen Zahl abgeleiteter Variablen (aus Darstellungsgründen 2 oder 3), aus denen sich die gemessenen Variablen nahezu ohne allzu großen Informationsverlust wiedergewinnen lassen. Es ist klar, dass man zu diesem Zweck die Korrelation zwischen den Variablen verwendet, die bei eNose-Daten im Allgemeinen hoch ist.

\section{Artifizielle Neuronale Netze (ANN)}

ANN sind durch ihre Lernfähigkeit gekennzeichnet. Angelehnt an das menschliche Gehirn, dessen Neuronen unzählige Verbindungen aufweisen, handelt es sich um Modellsysteme mit fiktiven, in Lagen angeordneten Neuronen und Verbindungen zwischen ihnen. Die Verbindungen sind durch mathematische Gewichtsfak- 


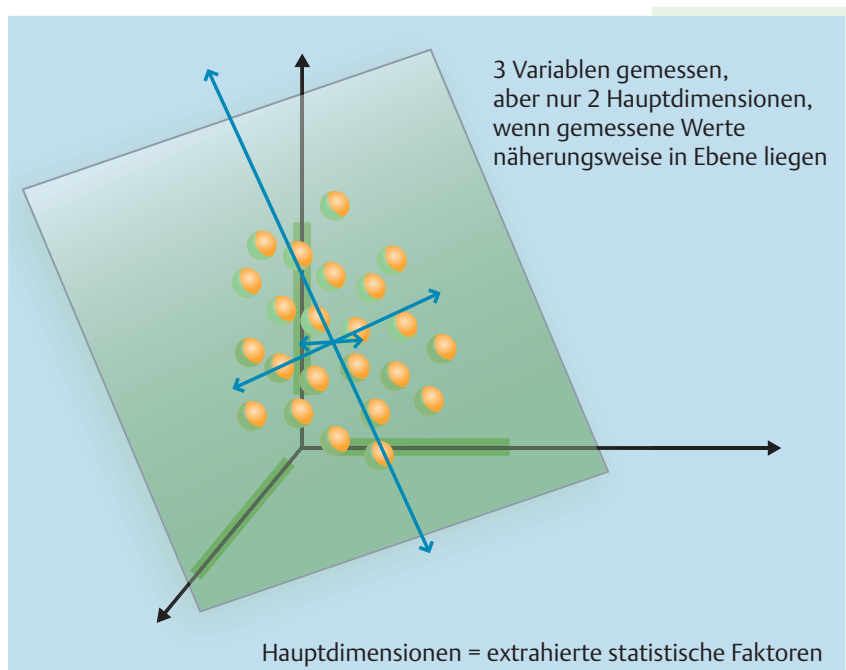

Abb. 2 Reduktion der Daten aus einer Vielzahl von Sensoren anhand ihrer Korrelation in wenige virtuelle Variablen, z. B. wie in dieser Grafik auf zwei Hauptdimensionen. Die Hauptdimensionen entsprechen der extrahierten Information.

toren charakterisiert und die Neuronen durch Schwellenwerte, bei deren Überschreitung sie aktiviert werden. Gibt man auf die Neuronenlage, welche an der Eingangsseite liegt, ein Muster von Signalen, reagieren die nachgeschalteten Neuronenlagen mit daraus abgeleiteten Aktivierungsmustern. Hierbei kann ein Neuron der am Ausgang befindlichen Neuronenlage beispielsweise eine klinische Entität repräsentieren, die es durch seine Aktivierung anzeigt. Die Gewichte der Verbindungen können mit Trainingssets durch ein kontrolliertes Lernverfahren in Trainingsläufen modifiziert werden mit dem Ziel, dass sich eine z. B. an dem klinischen Problem orientierte Ein-Ausgabe-Beziehung ergibt. Die Anwendung besteht darin, ein neues, unbekanntes Muster einem bestimmten Mustertyp zuzuordnen, der sich zuvor in einer gut definierten Krankheitsgruppe herausgestellt hat. $\mathrm{Zu}$ beachten ist, dass das Lernen immer auf eine bestimmte, vorher festgelegte Fragestellung orientiert ist und bei Veränderungen und Erweiterungen ein neuer Lernvorgang stattfinden muss.

Für alle Verfahren ist wichtig, die Relation zwischen der Anzahl der Probanden und der Anzahl der Messvariablen zu beachten. So kann man bei 600 Metaboliten in der Ausatemluft und 30 Probanden immer kombinatorisch solche Signale finden, die zwei Gruppen voneinander unterscheiden. Wird die Gruppe der Probanden größer, ergeben sich neue Signale zur Unterscheidung, oder aber die Unterschiede verwischen sich. Erst wenn die Zahl der Probanden, Variablen und Kategorien, in die eingeordnet werden soll, in vernünftiger Relation stehen, werden statistisch gesicherte und in der Einordnung verlässliche Aussagen möglich.

\section{Anwendungsgebiete der elektronischen Nase}

Elektonische Nasen werden vielfach im militärischen Bereich verwendet, um chemische und biologische Kampfstoffe zu detektieren. In der Lebensmittelchemie dienen sie der Qualitätskontrolle oder kontinuierlichen Überwachung der Lagerung von Lebensmitteln. Bislang liegen wenige Studien im medizinischen Bereich vor. So war es möglich, leichtgradig und schwergradig erkrankte Asthmatiker anhand des Signalmusters der Ausatemluft zu unterscheiden. Dies galt ebenfalls für den Vergleich gesunder Kon- trollpersonen und Asthmatiker [5]. Auch Patienten mit histologisch gesichertem, nicht-kleinzelligem Bronchialkarzinom und Patienten mit COPD wurden untersucht [6]. Interessant ist, dass es z. T. Probleme bereitete, die Signalmuster von Patienten mit COPD von denen von Rauchern zu unterscheiden [6,7]. Andererseits zeigten Patienten mit Lungenkrebs unterschiedliche Muster im Vergleich zu Gesunden unabhängig vom Raucherstatus, wobei hier die Diagnose vorher bekannt war [8]. Da man gute Argumente dafür finden kann, die Unterschiede zumindest teilweise auf systemische Veränderungen zurückzuführen, die sich in der Zusammensetzung der Ausatemluft widerspiegeln, ergeben sich interessante weitergehende Fragen, so etwa, ob die Analyse der Ausatemluft auch Hinweise auf Tumoren anderer Organe als der Lunge liefern kann oder, noch weiter gefasst, die elektronische Nase nicht ein diagnostisches oder Monitoring-Instrument für Systemerkrankungen sein kann.

\section{Spezielle Formen elektronischer Nasen \\ $\nabla$}

\section{Ionenmobilitätsspektrometrie (IMS)}

Prinzip der lonenmobilitätsspektrometrie

Unter Umgebungsdruck werden Ionen erzeugt, die sich in einem elektrischen Feld gegen die Strömungsrichtung eines Gases auf einer definierten Wegstrecke bewegen. Als Ergebnis des Feldes und der Zusammenstöße mit den Gasmolekülen erreichen die Ionen eine bestimmte mittlere Geschwindigkeit, die von der Masse und/oder chemischen Struktur abhängt. Der Quotient aus Ionengeschwindigkeit und elektrischer Feldstärke ist die Ionenmobilität; diese ist charakteristisch für die zu untersuchenden Analyte, sodass eine Identifikation des/der Analyte erfolgen kann. Meist wird zusätzlich eine gaschromatografische (GC) Vortrennung eingesetzt, sodass ein Analyt durch die Kombination von Driftzeit im IMS und Retentionszeit im GC identifiziert und quantifiziert werden kann ( $\bullet$ Abb. 3) [9].

Teilweise stehen bereits datenbankgestützte Verfahren zur Identifikation von Analyten bereit, die es erlauben, sowohl Zeitprofile zu ermitteln als auch größere Analytmengen systematisch zu untersuchen. Die Probenahme ist durch Kopplung mit einem Spirometer direkt mit einer Lungenfunktionsprüfung verknüpfbar, und die Probenahme kann entsprechend volumenkontrolliert erfolgen.

Aufbau des Ionenmobilitätsspektrometers ( $\bullet$ Abb. 3).

\section{Anwendung der lonenmobilitätsspektrometrie}

Neben der Anwendung zum Sprengstoffscreening an Flughäfen oder Aufgaben bei der Feuerwehr wird die IMS auch für wissenschaftliche Fragen in der Medizin eingesetzt. So konnte gezeigt werden, dass die Narkose mit Propofol über die Bestimmung von Metaboliten im Exhalat verfolgt werden kann und dass Patienten mit Sarkoidose oder mit Bronchialkarzinom typische Exhalatmuster aufweisen $[8,10]$.

\section{Massenspektrometrie}

$\nabla$

\section{Prinzip der Massenspektrometrie}

Die Massenspektrometrie ist ein Verfahren zum Messen geladener Ionen im Vakuum. Dazu wird die zu untersuchende Substanz in Gasform überführt und nachfolgend z. B. durch geringgradige radioaktive Strahlung ionisiert. Die ionisierten Teilchen werden durch ein elektrisches Feld beschleunigt und nach dem Verhältnis von Masse zu Ladung (negativer und positiver) aufgetrennt. 


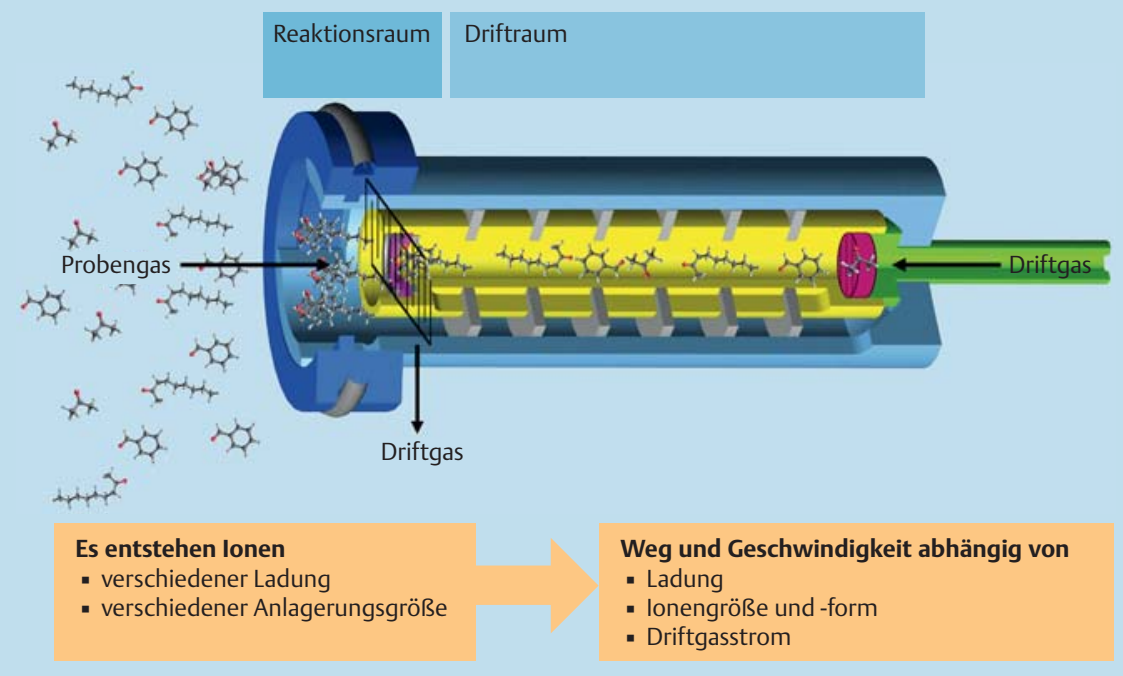

Abb. 3 In dieser Grafik wird das Grundprinzip der Ionenmobilitätsspektroskopie verdeutlicht. Im Reaktionsraum wird das Probengas eingeleitet. Es erfolgt die lonisierung. Der Weg und die Geschwindigkeit der lonen wird im Driftraum gemessen.

Das Massenspektrometer ist durch die beiden Parameter Massenauflösung und die Massengenauigkeit charakterisiert. Die Massenauflösung beschreibt den Massenunterschied, den zwei Ionen haben müssen, damit sie noch differenziert werden können. Die Massengenauigkeit beschreibt, wie genau die Masse des Ions bestimmt werden kann.

\section{Anwendung der Massenspektrometrie}

Es gibt unzählige Anwendungen in der analytischen Chemie (beispielsweise Bestimmung, wie häufig geladene Moleküle und deren Massenfragmente auftreten), Archäologie (Isotopenverhältnisse bei Knochenuntersuchungen), Biologie (Proteomik und Metabolomik), Klimatologie (Sedimente und Eisbohrkerne) oder Industrie (beispielsweise bei der Optimierung der Gaszufuhr bei Beschichtungen). Daneben findet die Massenspektrometrie Anwendung in der Urin- und Atemdiagnostik. In der Atemdiagnostik werden Atemwegskondensat, Sputum oder Lavageflüssigkeiten untersucht [11]. Zur Erhöhung der Empfindlichkeit wurden Anreicherungstechniken (SPME, solid phase micro extraction) entwickelt, wobei zuerst eine Sammlung durch Adsorption und danach eine thermische Desorption erfolgt $[12,13]$.

\section{Bewertung der neuen Technik vor dem Hintergrund schon verwendeter nicht-invasiver Techniken \\ $\nabla$}

Die elektronischen Nasen verfügen über eine hohe Nachweisgenauigkeit. Dies führt allerdings zu einer Zunahme von Stör- und Hintergrundsignalen und macht damit auch die Zuordnung der relevanten Signale schwieriger. Erste Erfolge bei der Differenzierung von Erkrankungsgruppen weckten die Hoffnung, dass derartige Verfahren die Phänotypisierung von Erkrankungen erleichtern könnten. Auch wenn hierbei noch viel grundlegende Arbeit notwendig ist, könnte dies gelingen, sofern präzise beschriebene Gruppen von Patienten zum Trainieren der Geräte herangezogen werden. In bisherigen Untersuchungen waren relativ kleine Fallzahlen, unterschiedliche Messtechniken mit dadurch bedingt nicht vergleichbaren Signalen sowie die komplexe Auswertung Hindernisse für eine breitere Akzeptanz und Anwendung. Vergleicht man das Verfahren der elektronischen Nase z. B. mit der NO-Messung, die für die Diagnose und das Monitoring vor allem eines Asthma allgemein als hilfreich gilt, so finden sich einige Parallelen. Hat man erhöhte NO-Werte gemessen, entsteht die Frage nach der diagnostischen bzw. therapeutischen Konsequenz; für das exhalierte NO liegen hierzu schon viele Daten vor. In ähnlicher Weise stellt sich die Frage nach der Bedeutung des durch die elektronische Nase zusätzlich erhobenen Befundes. Daher wird es bei der Auswertung darauf ankommen, klare Zuordnungen zu leisten. Um über die bereits etablierten Zuordnungen hinauszukommen, können dann bei entsprechend großen Datensätzen auch Verfahren der Clusteranalyse Verwendung finden, um vermeintlich klinisch vergleichbare Patienten anhand unterschiedlicher Muster zu separieren und sodann klinische Unterschiede dieser verschiedenen „Klassen“ zu eruieren.

Neben der Phänotypisierung von pulmonalen Erkrankungen können auch Aussagen zu systemischen Erkrankungen erfolgen. So wurde beispielsweise in einem Abstract gezeigt, dass Patienten mit Diabetes mellitus eine Korrelation des HbA1c und der EN-Signale zeigen [14]. Dies unterstreicht noch einmal die Einsatzmöglichkeit für systemische, nicht primär respiratorische Erkrankungen. Dies gilt ebenso für die Diagnostik des Lungenkarzinoms [6] oder anderer Karzinome.

\section{Ausblick}

Bislang haben die EN noch keinen Eingang in die klinische Routine gefunden, und ihre Anwendung ist auf wissenschaftliche Fragestellungen beschränkt. Die unterschiedlichen Verfahren haben jeweils Vor- und Nachteile, die zum Teil im Aufbau begründet sind. Es bleibt abzuwarten, welche Systeme sich für welche Fragestellungen als geeignet, $d$. h. sowohl praktikabel als auch hinreichend aussagekräftig, erweisen werden. Die Situation ist vielleicht analog zum alternativen Antrieb von Fahrzeugen, bei dem unterschiedliche Konzepte parallel entwickelt werden, eines Tages sich aber vermutlich ein Antriebskonzept durchsetzen wird. Aus diesem Grund müssen auch neutrale und wissenschaftlich fundierte Vergleichsmessungen und -bewertungen existieren, die möglicherweise für verschiedene Anwendungen zu unterschiedlichen Folgerungen kommen. Ungeachtet dessen bedarf es keiner visionären Vorstellungskraft, um diesen kommenden Techniken eine Zukunft vorherzusagen: Sie sind nicht-invasiv, bieten überschaubare Untersuchungskosten, schnelle Aussagen und eine potenziell weite Verbreitung bei vergleichsweise niedrigen Anschaffungskosten. Da bislang die Auswertung vor allem durch die unterschiedlichen Techniken, fehlende Standardisie- 
rungen sowie das Fehlen von optimierten Auswerte- und Lernalgorithmen erschwert ist, muss hier die Hauptarbeit geleistet werden.

\section{Interessenskonflikt}

Die Autoren geben an, dass kein Interessenkonflikt besteht.

\section{Institute}

${ }^{1}$ Klinik für Innere Medizin, Schwerpunkt Pneumologie, Philipps-Universität Marburg

${ }^{2}$ Abteilung Klinische Atemwegsforschung, Fraunhofer Institut für Toxikologie und Experimentelle Medizin, Hannover

3 Institut und Poliklinik für Arbeits-, Sozial- und Umweltmedizin, Ludwig-Maximilians-Universität München

${ }^{4}$ Abteilung Pneumologie, Department Innere Medizin, Universität Leipzig

${ }^{5}$ Abteilung Klinische Diagnostik, KIST Europe, Campus E71, Saarbrücken

\section{Literatur}

1 Lewis NS. Comparisons between mammalian and artificial olfaction based on arrays of carbon black-polymer composite vapor detectors. Acc Chem Res 2004; 37: 663 -672

2 Peris M, Escuder-Gilabert L. A 21st century technique for food control: electronic noses. Anal Chim Acta 2009; 638: 1-15

3 Marti MP, Boque R, Riu M et al. Fast screening method for determining 2,4,6-trichloroanisole in wines using a headspace-mass spectrometry (HS-MS) system and multivariate calibration. Anal Bioanal Chem 2003; 376: 497- 501

4 Marti MP, Mestres M, Sala C et al. Solid-phase microextraction and gas chromatography olfactometry analysis of successively diluted samples. A new approach of the aroma extract dilution analysis applied to the characterization of wine aroma. J Agric Food Chem 2003; 51: 7861 7865

5 Dragonieri S, Schot R, Mertens BJ et al. An electronic nose in the discrimination of patients with asthma and controls. J Allergy Clin Immunol 2007; 120: 856-862

6 Dragonieri S, Annema JT, Schot $R$ et al. An electronic nose in the discrimination of patients with non-small cell lung cancer and COPD. Lung Cancer 2009; 64: 166-170

7 Fens $N$, Zwinderman AH, van der Schee MP et al. Exhaled breath profiling enables discrimination of chronic obstructive pulmonary disease and asthma. Am J Respir Crit Care Med 2009; 180: 1076-1082

8 Westhoff $M$, Litterst P, Freitag $L$ et al. Ion mobility spectrometry for the detection of volatile organic compounds in exhaled breath of patients with lung cancer: results of a pilot study. Thorax 2009; 64: 744-748

9 Baumbach JI, Eiceman GA. Ion mobility spectrometry: arriving on site and moving beyond a low profile. Appl Spectrosc 1999; 53: 338A$355 \mathrm{~A}$

10 Westhoff $M$, Litterst $P$, Freitag $L$ et al. Ion mobility spectrometry in the diagnosis of sarcoidosis: results of a feasibility study. J Physiol Pharmacol 2007; 58 Suppl 5 (Pt 2): 739-751

11 Lin JL, Bonnichsen MH, Nogeh EU et al. Proteomics in detection and monitoring of asthma and smoking-related lung diseases. Expert Rev Proteomics 2010; 7: $361-372$

12 Mieth M, Kischkel S, Schubert JK et al. Multibed needle trap devices for on site sampling and preconcentration of volatile breath biomarkers. Anal Chem 2009; 81: 5851 - 5857

13 Mieth M, Schubert JK, Groger T et al. Automated needle trap heart-cut GC/MS and needle trap comprehensive two-dimensional GC/TOF-MS for breath gas analysis in the clinical environment. Anal Chem 2010; 82: $2541-2551$

14 Hofbauer J, Dressel H, Seissler J. Analyse der Ausatemluft mittels Elektronischer Nase bei Patienten mit Diabetes mellitus. Pneumologie 2010; 64: S206 